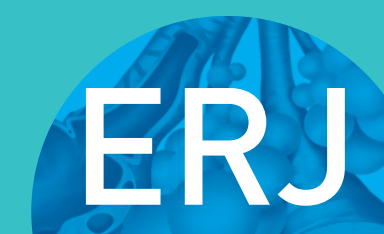

open research
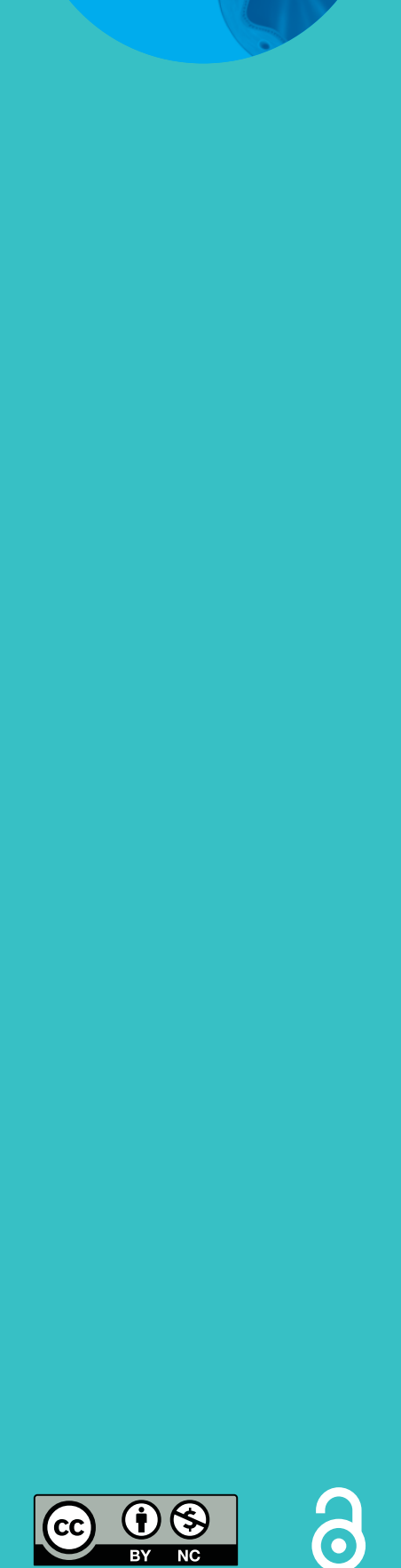

\section{Ventilator flow data predict bronchopulmonary dysplasia in extremely premature neonates}

\author{
Mariann H. Bentsen ${ }^{1,2}$, Trond Markestad ${ }^{1,2}$ and Thomas Halvorsen ${ }^{1,2}$
}

Affiliations: 'Dept of Pediatrics, Haukeland University Hospital, Bergen, Norway. ${ }^{2}$ Dept of Clinical Science, Faculty of Medicine and Odontology, University of Bergen, Bergen, Norway.

Correspondence: Mariann Bentsen, Dept of Pediatrics, Haukeland University Hospital, N-5021, Bergen, Norway. E-mail: benslahelse-bergen.no

ABSTRACT Early prediction of bronchopulmonary dysplasia (BPD) may facilitate tailored management for neonates at risk. We investigated whether easily accessible flow data from a mechanical ventilator can predict BPD in neonates born extremely premature (EP).

In a prospective population-based study of EP-born neonates, flow data were obtained from the ventilator during the first $48 \mathrm{~h}$ of life. Data were logged for $>10 \mathrm{~min}$ and then converted to flow-volume loops using custom-made software. Tidal breathing parameters were calculated and averaged from $\geqslant 200$ breath cycles, and data were compared between those who later developed moderate/severe and no/mild BPD.

Of 33 neonates, 18 developed moderate/severe and 15 no/mild BPD. The groups did not differ in gestational age, surfactant treatment or ventilator settings. The infants who developed moderate/severe BPD had evidence of less airflow obstruction, significantly so for tidal expiratory flow at $50 \%$ of tidal expiratory volume (TEF50) expressed as a ratio of peak tidal expiratory flow (PTEF) ( $\mathrm{p}=0.007)$. A compound model estimated by multiple logistic regression incorporating TEF50/PTEF, birthweight $\mathrm{z}$-score and sex predicted moderate/severe BPD with good accuracy (area under the curve 0.893, 95\% CI 0.735-0.973).

This study suggests that flow data obtained from ventilators during the first hours of life may predict later BPD in premature neonates. Future and larger studies are needed to validate these findings and to determine their clinical usefulness.

@ERSpublications

Bronchopulmonary dysplasia prediction from ventilator flow data http://ow.ly/uri130i0ZS9

Cite this article as: Bentsen $\mathrm{MH}$, Markestad T, Halvorsen T. Ventilator flow data predict bronchopulmonary dysplasia in extremely premature neonates. ERJ Open Res 2018; 4: 00099-2017 [https://doi.org/10.1183/23120541.00099-2017].

This study is registered at www.ClinicalTrials.com with identifier number NCT01150396.

Received: Aug 142017 | Accepted after revision: Jan 242018

Conflict of interest: None declared.

Support statement: The major funding institutions for this study were The Western Norway Regional Health Authority and the University of Bergen. Funding information for this article has been deposited with the Crossref Funder Registry.

Copyright $\odot$ ERS 2018. This article is open access and distributed under the terms of the Creative Commons Attribution Non-Commercial Licence 4.0. 


\section{Introduction}

Bronchopulmonary dysplasia (BPD) is the most common severe complication of premature birth and affects two out of three infants born extremely premature (EP), i.e. born before 28 weeks' gestational age [1]. It is associated with lifelong health consequences, including poor neurodevelopmental outcome and chronic respiratory conditions such as asthma, pulmonary hypertension and, possibly chronic obstructive pulmonary disease [2-4]. BPD is defined by need of oxygen supplementation for $\geqslant 28$ days and is graded as mild, moderate or severe depending on the requirement for oxygen supplementation or ventilatory support at 36 weeks' gestational age [5]. BPD is characterised by arrested lung growth with alveolar hypoplasia, abnormal pulmonary vasculature and interstitial cell proliferation [6] due to disruption of the normal in utero pulmonary development $[2,7,8]$. The disease process is multifactorial, but inflammation caused by various neonatal conditions, traumas and treatments is an essential driving force of the pathogenesis [9].

The risk of BPD is statistically linked to low gestational age, low birthweight and male sex [2, 4, 10], but EP-born neonates are probably born with variable susceptibilities or predispositions [4, 11]. We lack reliable clinical methods that might reflect such risk factors during the very early postnatal period; such methods are needed in order to improve our understanding of the disease process and thus provide a basis for prevention and targeted early management in neonates at particular risk. Our hypothesis was that early lung mechanics may reflect a susceptibility for later development of BPD, and our aim was to investigate whether flow data that are easily obtained from a ventilator during the first few hours of life can predict development of the severe forms of BPD in EP-born neonates in need of mechanical respiratory support.

\section{Methods}

Subjects and study design

This study was part of a larger prospective population-based cohort study (Project Extreme Prematurity; BabyPEP) that has been ongoing at Haukeland University Hospital (Bergen, Norway) since 2011. Pregnant females threatening preterm delivery before 28 weeks' gestation are invited and their children included if born $<28$ weeks gestational age. Since 2014, flow data have been logged from the ventilator for the neonates who received conventional ventilator support during their first $48 \mathrm{~h}$ of life.

\section{Ventilator flow data}

All neonates were ventilated using BabyLog VN500 (Dräger, Lübeck, Germany). Raw bidirectional flow data with a frequency of $50 \mathrm{~Hz}$ were logged for $>10$ min during the neonates' first $48 \mathrm{~h}$ of life using custom-made software (MedLink 4.4; Nortis Ingenieurbüro, Nürnberg, Germany) provided by the Dräger company. The software was installed on a computer connected to the ventilator by a Medibus cable. Flow-volume curves were constructed and breathing parameters analysed using another custom-made software package (VoluSense, Bergen, Norway). The mode of mechanical ventilation was "assist control with volume guarantee" during all data acquisitions. All neonates had uncuffed endotracheal tubes of sizes $2.5 \mathrm{~mm}(\mathrm{n}=31)$ or $3.0 \mathrm{~mm}(\mathrm{n}=2)$. The individual tube size was decided at the discretion of the attending neonatologist. All neonates were sedated and given morphine pain relief at doses $10-15 \mu \mathrm{g} \cdot \mathrm{kg}^{-1} \cdot \mathrm{h}^{-1}$; they were all clinically stable at the time of data acquisition, and laryngeal air leaks calculated by the ventilator were $<15 \%$.

\section{Analysis of flow-volume loops}

The analyses of the flow-volume loops and the selection of the traces upon which the analyses were based took place consecutively as the infants were included and before the patients were old enough to qualify for a BPD diagnosis, and thus were blinded to outcome. The traces were inspected visually to select $\geqslant 200$ stable breath cycles based on the following criteria: 1) no obvious artefacts; 2) no sighs; and 3) no obvious changes in the depth of breathing or baseline (figure 1a). The following breathing parameters were calculated and averaged by the computer software: tidal volume $(V \mathrm{~T})$, minute ventilation $\left(V^{\prime} \mathrm{E}\right)$, respiratory rate, peak tidal expiratory flow (PTEF), time to PTEF as a ratio of total expiratory time ( $t \mathrm{PTEF} / t \mathrm{E})$, ratio of tidal expiratory flow at $50 \%$ of expired volume (TEF50) to PTEF (figure 1b), ratio of tidal expiratory flow at $75 \%$ of expired volume (TEF75) to PTEF and flow volume gravity, which is a parameter described in detail previously [12]. We excluded $V \mathrm{~T}$, respiratory rate and $V^{\prime} \mathrm{E}$ from comparisons/statistical analyses, as these parameters primarily reflect support from the ventilator.

\section{Definitions}

BPD was defined according to the National Institute of Child Health and Human Development and National Heart, Lung, and Blood Institute workshop criteria from 2000, and the infants were retrospectively divided into two groups: no/mild BPD and moderate/severe BPD [5].

Z-scores for birthweights were calculated with reference to the 2013 Fenton growth charts [13]. 

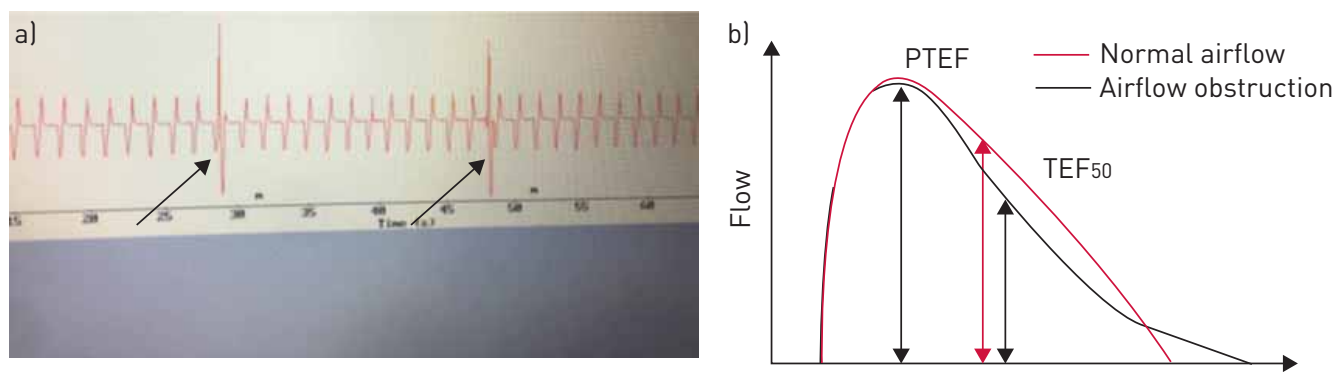

Tidal volume

FIGURE 1 Flow-volume loop analysis. a) Example of flow traces selected for computerised analysis. The arrows indicate breaths that were excluded from analysis (sighs). b) Airflow obstruction gives a concave flow-volume loop and lower tidal expiratory flow at 50\% of expired volume (TEF50)/peak tidal expiratory flow (PTEF) ratio.

\section{Statistical methods}

Data were analysed and graphs created using SPSS (version 22; IBM SPSS Statistics, Armonk, NY, USA) and MedCalc (version 13.1; MedCalc Software, Mariakerke, Belgium).

The data were tested for normal distribution, and means with standard deviations or $95 \%$ confidence intervals were calculated for the various patient variables and breathing parameters. Group comparisons were made with independent sample t-tests or the Chi-squared test, as appropriate. Receiver operator characteristic (ROC) analyses were used to assess the ability of the different expiratory breathing parameters, as well as a compound model consisting of TEF50/PTEF, birthweight z-score and sex, to predict BPD severity. As regards the compound model, a prognostic score (predicted probabilities) was estimated by multiple logistic regression, and this score was subsequently used as the test variable in the ROC analysis. Since gestational age and birthweight are highly correlated confounders, we chose to include birthweight $\mathrm{z}$-score in this model.

\section{Approvals}

The study was approved by the regional committee on medical research ethics of Western Norway (REC West) and registered at www.ClinicalTrials.gov (identifier number NCT01150396).

\section{Results}

\section{Subjects and perinatal data}

72 EP-born neonates were included in the BabyPEP study during the inclusion period (July 2014 to November 2016). 40 neonates received conventional ventilator support during their first $48 \mathrm{~h}$ of life, and 37 of them were included in this study. Flow data from three patients could not be recorded due to practical reasons. Data from four patients were excluded from the analyses due to death before 36 weeks gestational age preventing grading of BPD. Clinical characteristics and perinatal data for the remaining 33 patients are provided in table 1. 18 neonates developed moderate/severe BPD and 15 no/mild BPD. There were more males, and also a tendency for lower gestational age, birthweight and birthweight $\mathrm{z}$-score in the preterm-born neonates who developed moderate/severe BPD; however, differences were not statistically significant. Ventilatory support in terms of pressures and fractions of oxygen supplementation did not differ between the two BPD subgroups at the time of data acquisition.

\section{Comparisons between BPD subgroups and prediction of later BPD}

The moderate/severe BPD group had higher numerical values for all the expiratory breathing parameters than the no/mild BPD group, i.e. suggesting less obstruction, but the difference was only significant for TEF50/PTEF (table 2). This finding remained highly significant after performing a Bonferroni adjustment (adjusted p-value of 0.01).

ROC analyses showed that TEF50/PTEF predicted later development of moderate/severe BPD with an area under the curve (AUC) of 0.774 (95\% CI 0.596-0.901), but that gestational age (0.606, 0.421-0.771), birthweight $(0.672,0.487-0.825)$, birthweight $\mathrm{z}$-score or sex did not (figure 2$)$. The AUC (95\% CI) of a compound model incorporating TEF50/PTEF, birthweight z-score and sex was 0.893 (0.735-0.973). The optimal cut-off point for the combined model was 0.785 , meaning that a prognostic score $>0.785$ predicted BPD with sensitivity and specificity of $66.7 \%$ and $100 \%$, respectively, corresponding to positive and negative predictive values of $100 \%$ and $71.4 \%$ in this study. 


\section{TABLE 1 Perinatal characteristics of the extremely premature infants studied}

\begin{tabular}{|c|c|c|c|}
\hline & $\begin{array}{c}\text { Preterm-born neonates who } \\
\text { later developed } \\
\text { no/mild BPD }\end{array}$ & $\begin{array}{l}\text { Preterm-born neonates } \\
\text { who later developed } \\
\text { moderate/severe BPD }\end{array}$ & p-value ${ }^{\#}$ \\
\hline Subjects n & 15 & 18 & \\
\hline Male & $7(47)$ & $11(61)$ & 0.65 \\
\hline Gestational age at birth weeks ${ }^{\text {days }}$ & $26^{2} \pm 1^{1}$ & $25^{5} \pm 1^{3}$ & 0.24 \\
\hline Birthweight g & $864 \pm 164$ & $754 \pm 154$ & 0.06 \\
\hline Birthweight z-scores & $0.11 \pm 0.54$ & $-0.29 \pm 0.67$ & 0.08 \\
\hline Antenatal steroids & 13 (87) & $17(94)$ & 0.93 \\
\hline Surfactant treatment & $15(100)$ & $18(100)$ & 1.00 \\
\hline Age at data acquisition $\mathrm{h}$ & $17.8 \pm 13.3$ & $17.1 \pm 12.4$ & 0.87 \\
\hline PIP १ $\mathrm{CmH}_{2} \mathrm{O}$ & $16.1 \pm 2.3$ & $16.8 \pm 4.2$ & 0.55 \\
\hline PEEP १ $\mathrm{cmH}_{2} \mathrm{O}$ & $4.9 \pm 0.48$ & $4.8 \pm 0.62$ & 0.74 \\
\hline Set tidal volume per $\mathbf{k g}{ }^{\text {? }} \mathbf{m L} \cdot \mathbf{k g}^{-1}$ & $5.4 \pm 0.78$ & $5.2 \pm 0.66$ & 0.34 \\
\hline Fraction of inspired oxygen $\%$ & $32 \pm 15$ & $28 \pm 10$ & 0.40 \\
\hline
\end{tabular}

Data are presented as $\mathrm{n}(\%)$ or mean $\pm \mathrm{SD}$, unless otherwise stated. BPD: bronchopulmonary dysplasia; PIP: ventilator peak inspiratory pressure; PEEP: ventilator positive end-expiratory pressure. \#: independent samples t-test or Chi-squared test; ${ }^{\text {ๆ: }}$ during data acquisition.

\section{Discussion}

The expiratory flow ratio TEF50/PTEF obtained during the first $48 \mathrm{~h}$ of life was significantly higher, i.e. suggesting a less obstructive airflow pattern, for the EP-born neonates who later went on to develop moderate/severe BPD than for those who developed no or mild BPD. In addition, the TEF50/PTEF ratio significantly predicted moderate/severe $\mathrm{BPD}$, in contrast to other neonatal variables such as gestational age, birthweight and birthweight z-score. A compound model estimated by multiple logistic regression incorporating TEF50/PTEF ratio, birthweight $\mathrm{z}$-score and sex predicted moderate/severe BPD with good accuracy (AUC 0.893).

\section{Strengths and limitations}

Like any single-centre study with relatively few participants, the results of this study must be interpreted cautiously, as features particular to the participants or institutional practices may have introduced bias difficult to control for. The likelihood of selection bias was reduced to the extent possible by the inclusion procedure, i.e. convenience based and consecutive recruitment with few dropouts from a population-based sample of EP-born neonates. We only studied EP-born neonates who required early ventilatory support, and neonates with relatively little lung disease shortly after birth may still develop BPD [14]. Obviously, other prognostic methods are needed for this group, and electromagnetic inductance plethysmography is emerging as a promising method in this respect, since it can be applied in patients needing continuous positive airway pressure or high-flow nasal cannulas [15-17].

TABLE 2 Expiratory breathing characteristics calculated from flow data from a mechanical ventilator during the first $48 \mathrm{~h}$ of life in 33 extremely premature infants with or without later development of moderate/severe bronchopulmonary dysplasia (BPD)

Preterm-born neonates
later developing no/mild BPD

Subjects $\mathbf{n}$
PTEF per $\mathbf{k g ~ m L} \cdot \mathbf{s}^{-1}$
tPTEF/tE
TEF50/PTEF \%
TEF75/PTEF \%
FV

15

$27.6(22.1-33.2)$

$21.1(15.7-26.5)$

$71.8(62.9-80.6)$

$44.1(33.7-54.5)$

$0.42(0.39-0.44)$
Preterm-born neonates later developing moderate/severe BPD

Data are presented as mean $(95 \% \mathrm{CI})$, unless otherwise stated. $\mathrm{PTTF}$ : time to peak tidal expiratory flow; $t \mathrm{E}$ : expiratory time; TEFn: tidal expiratory flow at $n \%$ of expired volume; PTEF: peak tidal expiratory flow; FV: flow volume gravity. ${ }^{\#}$ : independent samples t-test. 


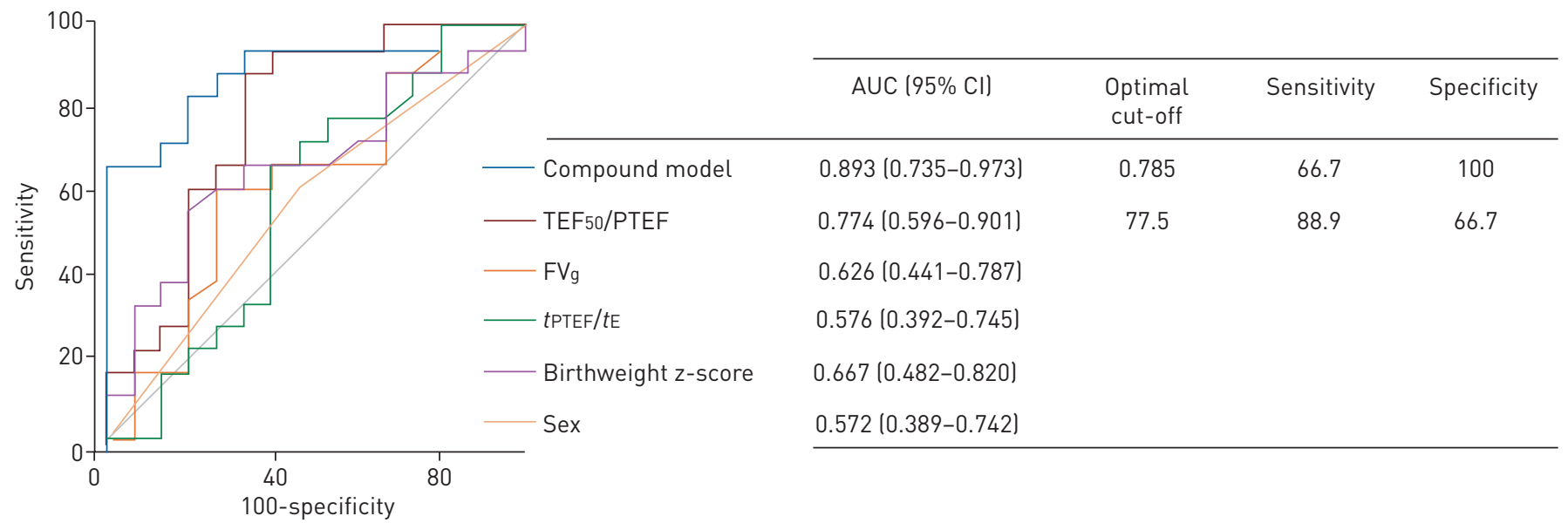

FIGURE 2 Receiver operator characteristic (ROC) curves comparing the ability of different expiratory breathing parameters and perinatal variables (birthweight $z$-score and sex) to predict development of bronchopulmonary dysplasia (BPD) ( $n=33$ ). The combination model incorporates tidal expiratory flow at $50 \%$ of expired volume (TEF50) to peak tidal expiratory flow (PTEF) ratio, birthweight z-score and sex. If the $95 \%$ confidence interval of the area under the ROC curve (AUC) includes 0.5 (no discrimination) the parameter does not predict BPD. The optimal cut-off point is where the sensitivity and specificity are maximal. FVg: flow volume gravity; $t$ PTEF: time to peak tidal expiratory flow; tE: expiratory time.

We cannot exclude that our findings reflect flow conditions or secretions in the endotracheal tubes. However, there were no clinical signs of tube obstruction in any of the participants during the time of data acquisition, and all neonates had an appropriately sized tube with minimal laryngeal air leaks. Furthermore, the obtained expiratory flow patterns do not agree with the pattern expected with tube obstructions or leaks. Expiration is primarily a passive process, and expiratory breathing parameters were thus considered to reflect natural lung mechanics. The positive end-expiratory pressure provided by the ventilator could theoretically bias the data, but it was almost identical in the two BPD subgroups.

\section{Comparisons between the two subgroups of BPD}

The neonates who went on to develop moderate/severe BPD had the least obstructive airflow pattern in the first few hours of life. Only the variable describing mid-expiratory flow (TEF50/PTEF) differed significantly between the two groups of $\mathrm{BPD}$, but the overall pattern was strengthened by the nonsignificant but coherent group differences also for the other expiratory flow parameters. In contrast, $\mathrm{BPD}$ in preterm-born infants is usually associated with increased expiratory airflow obstruction at term-equivalent age as well as throughout childhood, adolescence and early adulthood [18-20]. We do not have data to explain this unexpected finding. However, it is not unreasonable to assume that those neonates who later went on to develop the more severe forms of BPD were those who were born with the most immature lungs, characterised by low compliance due to low surfactant production and less developed and stiffer small and medium sized airways and distal respiratory airway structures [21, 22]. This is in accordance with major studies of EP-born neonates which show that low gestational age (i.e. increasing degree of prematurity) is a risk factor for severe BPD development within this already low gestational age group [23, 24]. It may further be speculated that this scenario could lead to more intense early interstitial and peribronchial inflammatory responses to positive pressure ventilation with a further decrease in lung and airway compliance. Additionally, the chest wall has a major influence on the underlying lungs, as its outward recoil is generally extremely low in preterm-born infants because of the soft rib cage and scarce intercostal muscles [22]. The overall effect for the most severely ill neonates could be an initial phase characterised primarily by high pulmonary elastic recoil pressures and reduced compliance, creating tidal airflow patterns that are less obstructive. This line of thinking is compatible with the clinically well recognised concept of "stiff lungs"; a term often used to describe the breathing pattern observed in the most severely ill preterm-born neonates with the more severe forms of neonatal respiratory distress syndrome.

Several studies suggest that neonates who go on to develop different severity of BPD have innate lung differences. Those who develop the severe forms tend to need more prolonged mechanical ventilation [20, 25], a larger proportion are treated with surfactant, they have different cytokine and growth factor patterns $[26,27]$ and a significant genetic predisposition has been proposed, although it is poorly understood $[4,11]$.

\section{Prediction of later BPD from early airflow parameters}

To our knowledge, the present study is the first attempt to examine to what extent easily accessible flow data from a mechanical ventilator can be used to predict later development of BPD in EP-born neonates. 
BHUTANi et al. [28] studied the relationship between pulmonary compliance and resistance and subsequent BPD in low birthweight infants $(\leqslant 1500 \mathrm{~g})$ who required mechanical ventilation during their first week of life, and found that low dynamic pulmonary compliance $(C \mathrm{~L})$ and high total pulmonary resistance were related to BPD. In addition, they created different BPD prediction models and found that a model dependent on gestational age and CL had the highest positive predictive accuracy. KIM et al. [29] found that modified respiratory parameters, i.e. peak inspiratory pressure relative to birthweight and mean airway pressure relative to birthweight at $12 \mathrm{~h}$ of age were significant risk factors for the development of BPD.

Various scoring systems for predicting later BPD based on clinical variables such as gestational age, birthweight, sex, fraction of inspired oxygen, exposure to mechanical ventilation, sepsis and presence of patent ductus arteriosus have been proposed, but generally they have not been adopted in clinical practice. A general problem of these scoring systems has been their relatively low specificity or low positive predictive values [30]. Furthermore, the need for prolonged observation and inclusion of unpredictable complications obscure the significance of innate predispositions which, in our opinion, is fundamental in an attempt to understand probable causes and thus ways of preventing a progression towards severe chronic lung disease in children born prematurely.

\section{Conclusions}

Flow data easily obtained from a ventilator could be used to compute breathing parameters that discriminated between neonates who went on to develop the severe forms of BPD and those who did not. The parameter TEF50/PTEF showed promising predictive abilities in this respect, and a model combining this parameter with birthweight $\mathrm{z}$-score and sex could predict later respiratory morbidity with good accuracy. Future and larger studies are needed to validate these findings and to determine their clinical usefulness. If subsequent evaluations were to confirm the findings, this would open for targeted management and institution of customised preventive and early therapeutic measures in neonates at particular risk of developing severe BPD.

\section{Acknowledgements}

The authors thank Morten Eriksen (VoluSense, Bergen, Norway) for helpful technical assistance, and Geir Egil Eide (Center for Clinical Research, Haukeland University Hospital, Bergen, Norway) for help with statistical methods and interpretation. Last but not least, we thank the infants who participated in this study and their parents.

\section{References}

1 Stoll BJ, Hansen NI, Bell EF, et al. Neonatal outcomes of extremely preterm infants from the NICHD Neonatal Research Network. Pediatrics 2010; 126: 443-456.

2 Baraldi E, Filippone M. Chronic lung disease after premature birth. N Engl J Med 2007; 357: 1946-1955.

3 Vollsæter M, Røksund OD, Eide GE, et al. Lung function after preterm birth: development from mid-childhood to adulthood. Thorax 2013; 68: 767-776.

4 Bhandari A, Bhandari V. Pitfalls, problems, and progress in bronchopulmonary dysplasia. Pediatrics 2009; 123: $1562-1573$.

5 Jobe AH, Bancalari E. Bronchopulmonary dysplasia. Am J Respir Crit Care Med 2001; 163: 1723-1729.

6 Coalson JJ. Pathology of bronchopulmonary dysplasia. Semin Perinatol 2006; 30: 179-184

7 Baker CD, Alvira CM. Disrupted lung development and bronchopulmonary dysplasia: opportunities for lung repair and regeneration. Curr Opin Pediatr 2014; 26: 306-314.

8 Stocks J, Hislop A, Sonnappa S. Early lung development: lifelong effect on respiratory health and disease. Lancet Respir Med 2013; 1: 728-742.

9 Speer CP. Chorioamnionitis, postnatal factors and proinflammatory response in the pathogenetic sequence of bronchopulmonary dysplasia. Neonatology 2009; 95: 353-361.

10 Jobe AH. The new bronchopulmonary dysplasia. Curr Opin Pediatr 2011; 23: 167-172.

11 Yu KH, Li J, Snyder M, et al. The genetic predisposition to bronchopulmonary dysplasia. Curr Opin Pediatr 2016; 28: 318-323.

12 Bentsen MH, Haaland ØA, Lønning LB, et al. A new non-invasive method of infant spirometry demonstrates a level of repeatability that is comparable to traditional methods. Acta Paediatr 2015; 104: 1130-1137.

13 Fenton TR, Kim JH. A systematic review and meta-analysis to revise the Fenton growth chart for preterm infants. BMC Pediatr 2013; 13: 59 .

14 Jobe AH. What is BPD in 2012 and what will BPD become? Early Hum Dev 2012; 88: Suppl. 2, S27-S28.

15 Pickerd N, Williams EM, Kotecha S. Electromagnetic inductance plethysmography to measure tidal breathing in preterm and term infants. Pediatr Pulmonol 2013; 48: 160-167.

16 Pickerd N, Williams EM, Watkins WJ, et al. Tidal breathing in preterm infants receiving and weaning from continuous positive airway pressure. J Pediatr 2014; 164: 1058-1063.

17 Bentsen $\mathrm{MH}$, Eriksen M, Olsen MS, et al. Electromagnetic inductance plethysmography is well suited to measure tidal breathing in infants. ERJ Open Res 2016; 2: 00062-2016.

18 Olden C, Symes E, Seddon P. Measuring tidal breathing parameters using a volumetric vest in neonates with and without lung disease. Pediatr Pulmonol 2010; 45: 1070-1075.

19 Schmalisch G, Wilitzki S, Wauer RR. Differences in tidal breathing between infants with chronic lung diseases and healthy controls. BMC Pediatr 2005; 5: 36.

20 Vollsæter M, Skromme K, Satrell E, et al. Children born preterm at the turn of the millennium had better lung function than children born similarly preterm in the early 1990s. PLoS One 2015; 10: e0144243. 
21 Strang LB. The lungs at birth. Arch Dis Child 1965; 40: 575-582.

22 Hammer J, Eber E, eds. Paediatric Pulmonary Function Testing. Progress in Respiratory Research. Vol. 33. Basel, Karger, 2005; pp. 2-7.

23 Laughon M, Langer JC, Bose C, et al. Prediction of bronchopulmonary dysplasia by postnatal age in extremely premature infants. Am J Respir Crit Care Med 2011; 183: 1715-1722.

24 Marshall DD, Kotelchuck M, Young TE, et al. Risk factors for chronic lung disease in the surfactant era: a North Carolina population-based study of very low birth weight infants. Pediatrics 1999; 104: 1345-1350.

25 Hjalmarson O, Sandberg KL. Lung function at term reflects severity of bronchopulmonary dysplasia. J Pediatr 2005; 146: 86-90.

26 Schneibel KR, Fitzpatrick AM, Ping XD, et al. Inflammatory mediator patterns in tracheal aspirate and their association with bronchopulmonary dysplasia in very low birth weight neonates. J Perinatol 2013; 33: 383-387.

27 Bose CL, Dammann CE, Laughon MM. Bronchopulmonary dysplasia and inflammatory biomarkers in the premature neonate. Arch Dis Child Fetal Neonatal Ed 2008; 93: F455-F461.

28 Bhutani VK, Abbasi S. Relative likelihood of bronchopulmonary dysplasia based on pulmonary mechanics measured in preterm neonates during the first week of life. J Pediatr 1992; 120: 605-613.

29 Kim YD, Kim EA, Kim KS, et al. Scoring method for early prediction of neonatal chronic lung disease using modified respiratory parameters. J Korean Med Sci 2005; 20: 397-401.

30 Trembath A, Laughon MM. Predictors of bronchopulmonary dysplasia. Clin Perinatol 2012; 39: 585-601. 